\title{
LATTICE DYNAMICS OF COMMENSURATE MONOLAYERS ADSORBED ON GRAPHITE
}

\author{
H. J. LAUTER $\$$, V. L. P. FRANK $\S^{*}$, H. TAUB ${ }^{+}$and P. LEIDERER ${ }^{*}$ \\ § Institute Laue-Langevin, BP 156X, F-38042 Grenoble, France \\ * University of Konstanz, D-7750 Konstanz 1, West Germany \\ + University of Missouri-Columbia, Columbia, Missouri 65211, USA
}

\begin{abstract}
A review of the lattice dynamics of gases adsorbed on graphite in the commensurate phase is presented, taking into account their quantum character. The phonon dispersion curves are characterized by an energy gap in the acoustic branch at the zone center. Its magnitude is related to the corrugation of the in-plane adsorption potential. The energy of the zone boundary phonons, on the other hand, is determined by the interaction between adatoms. The measurement of these quantities allows the comparison with theoretical models.
\end{abstract}

\section{INTRODUCTION}

During the last decade, substantial progress in the knowledge of the properties of two-dimensional (2D) matter has been achieved thanks to studies done on monolayers of gases adsorbed on well characterized substrates like graphite. The substrate can impose its periodicity on the position of the adsorbed atoms, often leading to a commensurate structure. In this phase, the lack of translational invariance produces a gap $(\Delta)$ in the acoustic branch at the zone center of the phonon dispersion relation. The magnitude of $\Delta$ is related to the corrugation of the adsorption potential. Changes in temperature and/or coverage induce phase transitions that are the result of a delicate balance between the interaction of the adsorbed gas and the substrate, and between the adsorbed gas molecules themselves. Unfortunately, the knowledge of the details of these interaction potentials is sparse, since not many experimental techniques are available. The adsorption potential itself has been determined mainly with molecular beam scattering, but the magnitude of the inplane corrugation of the adsorption potential is very difficult to obtain in this way. The temperature renormalization of the phonon spectrum gives insight into the anharmonic terms of the adsorption and intermolecular potentials. Recently, several measurements of the gap became available (1-8) and allowed a comparison with theoretical models of the adsorption potentials.

\section{EXPERIMENTAL RESULTS}

We present here a review of results of inelastic neutron scattering experiments that determined $\Delta$ and its dependence on temperature, for various substances. The studied adsorbates present all a registered $(\sqrt{3 * \sqrt{ }}) \mathrm{R} 30^{\circ}$ phase $\left(a_{n n}=4.26 \AA\right)$ and can be grouped mainly into two classes according to their quantum character (see Table I). The hydrogen isotopes $\left(\mathrm{H}_{2}, \mathrm{HD}, \mathrm{D}_{2}\right)(1-3)$ and ${ }^{3} \mathrm{He}(4)$ are typical quantum gases: their interaction potential is weak, they exhibit a large zero point motion and a very large compressibility (the commensurate $a_{n n}$ is much larger than the 3D-solid one). Nitrogen $\left(\mathrm{N}_{2}\right)(5,6)$, deuterated-methane (CD4) (7) and Krypton (8), on the other hand, are much heavier molecules/atoms, with consequently a smaller zero point motion forming less compressible monolayers. In these cases the $3 \mathrm{D}$ lattice parameter matches within some percent the one of the $2 \mathrm{D}$ commensurate phase.

Table I: Parameters that characterize the adsorbate gas - graphite system in the commensurate phase, the adsorbates are ordered with increasing de Boer parameter.

a the de Boer parameter indicates the quantum character of a substance, $\Lambda=h / \sigma \sqrt{ }(m \varepsilon)$, it is the ratio between the de Broglie wavelength of the relative motion of two atoms with energy $\varepsilon$ and the minimum in the LJ potential.

b This column presents the width of the in-plane phonon density of states (DOS). These values are hard to determine with inelastic neutron scattering, since the intensity of the structure factor decreases with increasing energy.

c The phase diagrams of $\mathrm{Kr}, \mathrm{N}_{2}$ and $\mathrm{CD}_{4}$ present a commensurate region that extends to higher temperatures when the coverage is slightly higher than the commensurate one. Details of the phase diagram can be found in refs. (9), (10) and (11), respectively.

\begin{tabular}{|c|c|c|c|c|c|c|c|c|}
\hline \multirow[t]{2}{*}{ Adsorbate } & \multirow{2}{*}{$\begin{array}{l}\text { Mass } \\
{[\text { a.u.] }}\end{array}$} & \multirow{2}{*}{$\begin{array}{l}\text { de Boer }{ }^{a} \\
\text { parameter }\end{array}$} & \multicolumn{2}{|c|}{ Lennard-Jones parameter } & \multirow{2}{*}{$\begin{array}{c}\text { Gap energy } \\
{[\mathrm{K}]}\end{array}$} & \multirow{2}{*}{$\begin{array}{c}\text { Gap ratio } \\
\Delta_{\text {mead }} / \Delta_{\text {calc }}\end{array}$} & \multirow{2}{*}{$\begin{array}{c}\text { DOS widthb } \\
{[\mathrm{K}]}\end{array}$} & \multirow{2}{*}{$\begin{array}{c}\text { Melting } \\
\text { Temperature }\end{array}$} \\
\hline & & & $\varepsilon[\mathrm{K}]$ & $\sigma[\AA]$ & & & & \\
\hline $\mathrm{Kr}$ & 83.8 & 0.10 & 165.3 & 3.63 & 8.7 & 1,0 & - & $\sim 125^{\mathrm{c}}$ \\
\hline $\mathrm{CD}_{4}$ & 20.0 & 0.23 & 137.0 & 3.68 & 14.5 & 0,9 & 48 & $\sim 55^{\mathrm{c}}$ \\
\hline $\mathrm{N}_{2}$ & 28.0 & 0.42 & 35.6 & 3.32 & 19.3 & 1,7 & 34 & $\sim 72^{\mathrm{C}}$ \\
\hline $\mathrm{D}_{2}$ & 4.0 & 1.26 & 35.2 & 2.95 & 40.0 & $\overline{1,1}$ & 9.5 & 18.5 \\
\hline HD & $3.0^{-}$ & 1.43 & 35.9 & 2.95 & 43.2 & 1,1 & 14.7 & 19.4 \\
\hline $\mathrm{H}_{2}$ & 2.0 & 1.74 & 36.7 & 2.95 & 47.3 & 1,0 & 27.5 & 20.5 \\
\hline${ }^{3} \mathrm{He}$ & 3.0 & 3.10 & 10.2 & 2.56 & 10.9 & 0,3 & 40 & 3.05 \\
\hline
\end{tabular}


One of the most striking differences between both types of adsorbates is the temperature dependence of the gap energy. On a flat substrate no gap exists, and the acoustic phonon branches go to zero at the zone center. In this case, a non-zero temperature produces thermal phonons. In the case of a commensurate phase, the acoustic phonon branches have a finite value at the zone center: the phonon gap. In order to thermally populate phonon modes a minimum temperature has to be reached (of the order of the gap energy). At lower temperatures mainly the zero point vibration remains. All the quantum gases melt at a temperature $\left(\mathrm{T}_{\mathrm{m}}\right)$ that is between $30-50 \%$ of the energy of the gap: at $\mathrm{T}_{\mathrm{m}}$ a negligible fraction of thermal phonons are excited. Thus, a small increase in $\left.\sqrt{ }<u^{2}\right\rangle$ (12) due to these thermal phonons suffices to melt the commensurate monolayer. This increase occurs at a temperature that is very close to $T_{m}$. As a consequence, the gap energy does not change up to $\mathrm{T}_{\mathrm{m}}$ and the phonon spectrum is practically not affected by temperature. On the other hand, the heavier gases melt at a temperature that is much higher (at least a factor 3) than the gap energy and a strong temperature renormalization of the phonon spectrum occurs $(13,14)$. This can bee seen in figure 1 , where the data for the phonon gap as a function of temperature are depicted for several gases.

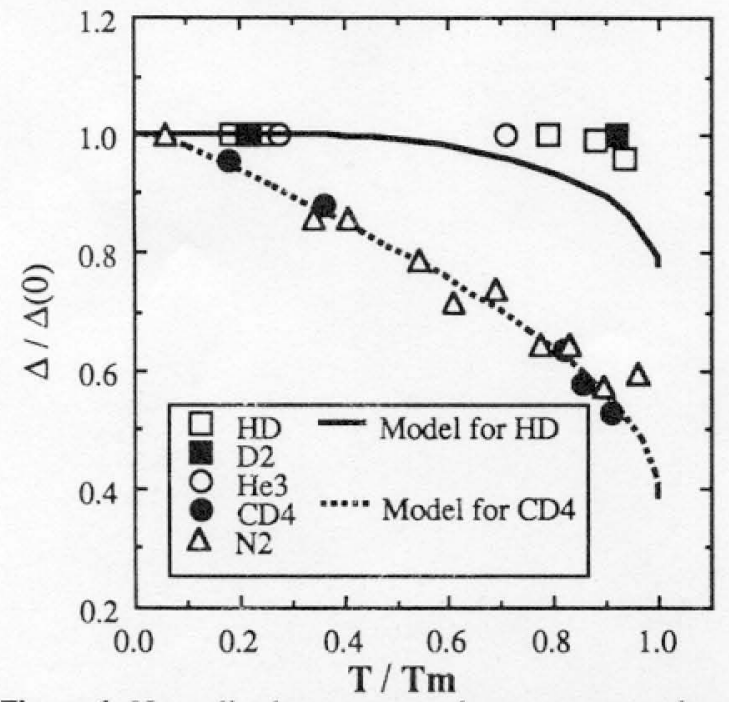

Figure 1: Normalized zone center phonon gap vs. reduced temperature for several gases adsorbed on graphite. The lines are the result of the model calculation described in ref. 13. ( $\Delta$ stands for the zone center phonon gap)

The agreement between theoretical calculations of $\Delta$ $(15,16)$ and the measured values is indicated in the $7^{\text {th }}$ column of Table I. Two elements present a large deviation from the expected magnitude: $\mathrm{N}_{2}$ and ${ }^{3} \mathrm{He}$. ${ }^{3} \mathrm{He}$ is particularly hard to model due to its high quantum character that makes it very difficult to take into account all the correlations and many body effects (16). A single ${ }^{3} \mathrm{He}$ atom does not stay on an adsorption site, but needs the presence of the other adatoms to localize. Already $\mathrm{H}_{2}$ localizes at low coverages and the calculation (15) agrees well with experiment. For $\mathrm{N}_{2}$ no simple explanation for this disagreement has been found and this suggests that further work is required to improve our knowledge of the adatom-substrate potential (5).

\section{ACKNOWLEDGEMENTS}

This work has been partially supported by the Federal Ministry of Research and Technology (BMFT) of the Federal Republic of Germany.

\section{REFERENCES}

(1) Frank V.L.P., Lauter H.J. and Leiderer P., Jpn.J.Appl.Phys.Suppl. 26, 347 (1987)

(2) Frank V.L.P., Lauter H.J. and Leiderer P., Phys.Rev.Lett., 61, 436 (1988)

(3) Lauter H.J., Frank V.L.P., Leiderer P. and Wiechert H., Physica B, 156\&157, 280 (1989)

(4) Frank V.L.P., Lauter H.J., Godfrin H. and Leiderer P., PHONONS 89, pp. 1001 (World Scientific, Singapore, 1990)

(5) Hansen F.Y., Frank V.L.P., Taub H., Bruch L.W. Lauter H.J. and Dennison J.R., Phys.Rev.Lett., 64, 764 (1990)

(6) Frank V.L.P., Lauter H.J., Hansen F.Y., Taub H., Bruch L.W. and Dennison J.R., PHONONS 89, pp.922 (World Scientific, Singapore, 1990)

(7) Moeller T., Lauter H.J., Frank V.L.P. and Leiderer P., PHONONS 89, pp.919 (World Scientific, Singapore, 1990)

(8) Frank V.L.P., Lauter H.J. and Taub H., unpublished data.

(9) Butler D.M., Litzinger J.A. and Stewart G.A., Phys.Rev.Lett. 44, 466 (1980)

(10) Chan M.H.W., Migone A.D., Miner K.D. and Li Z.R., Phys.Rev.B, 30, 2681 (1984)

(11) Kim H.K., Zhang Q.M., Chan M.H.W., Phys.Rev.B, 34, 4699 (1986)

(12) The rms vibrational amplitude $\left(\sqrt{ }<u^{2}>\right)$ at a given temperature can be expressed as a thermally weighted average over the phonon density of states (DOS).

(13) Frank V.L.P., Lauter H.J., Godfrin H. and Leiderer P., PHONONS 89, pp.913 (World Scientific, Singapore, 1990)

(14) Hakim T.M., Glyde H.R. and Chui S.T., Phys.Rev.B, 37, 974 (1988)

(15) Novaco A.D., Phys.Rev.Lett., 61, 436 (1988)

(16) Ni X.-Z. and Bruch L.W., Phys.Rev.B, 33, 4584 (1986) 